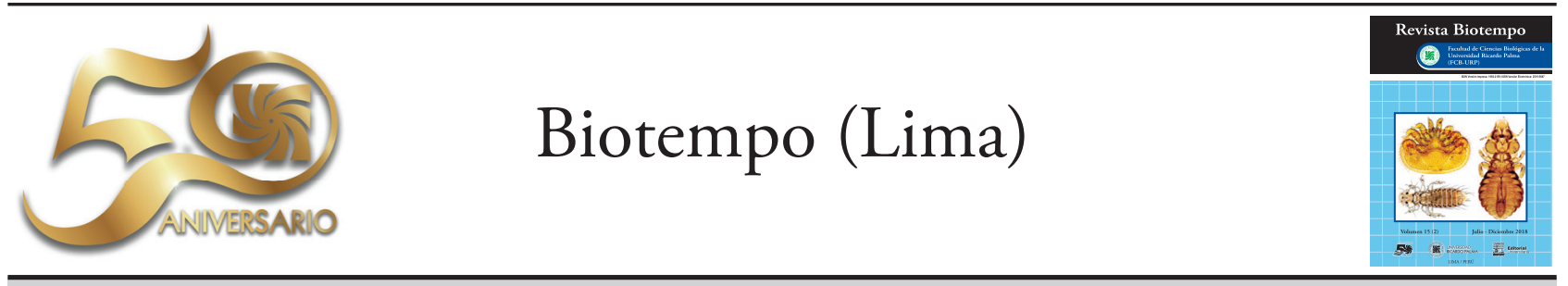

ORIGINAL ARTICLE / ARTÍCULO ORIGINAL

ACID OPTIMIZATION FOR THE ECOTOXICOLOGICAL BIOEXTRACTION OF METALS IN TARGET ORGANS

\title{
OPTIMIZACIÓN ÁCIDA PARA LA BIOEXTRACCIÓN ECOTOXICOLÓGICA DE METALES EN ÓRGANOS DIANAS
}

Humberto Argota-Coello ${ }^{1}$; George Argota-Pérez ${ }^{2}$; José Iannacone ${ }^{3,4} \&$ Rigoberto Fimia-Duarte $^{5}$

1 Laboratorio de Minerales. Empresa Geominera Oriente. Santiago de Cuba, Cuba. h.argota.coello@gmail.com

2 Centro de Investigaciones Avanzadas y Formación Superior en Educación, Salud y Medio Ambiente "AMTAWI", Puno-Perú.george.argota@gmail.com

3 Laboratorio de Parasitología. Facultad de Ciencias Biológicas. Universidad Ricardo Palma (URP). Lima-Perú. joseiannacone@gmail.com

4 Laboratorio de Ecología y Biodiversidad Animal. Facultad de Ciencias Naturales y Matemática. Universidad Nacional Federico Villarreal (UNFV). Lima-Perú;

5 Facultad de Tecnología de la Salud "Julio Trigo López". Universidad de Ciencias Médicas de Villa Clara, Cuba. rigobertofd@infomed.sld.cu

Author for correspondence: george.argota@gmail.com

\section{ABSTRACT}

The purpose of the study was to optimize an acid mixture for the ecotoxicological bioextraction of metals in target organs. From June 2010 to June 2017, six types of acids were selected: $\mathrm{HF}, \mathrm{HCl}, \mathrm{HNO}_{3}, \mathrm{HClO}_{4}, \mathrm{H}_{2} \mathrm{SO}_{4}$ and $\mathrm{H}_{3} \mathrm{PO}_{4} .15$ combinations (two types of acids) and 17 combinations (three types of acids) were designed to compare their extraction e with the standard reference values certified on fish not exposed to metal contamination: DOLT-3 ("Dogfish Liver", shark liver) and DORM-2 ("Dogfish Muscle", shark muscle). The closest registers corresponded to the mixture: $\mathrm{HNO}_{3}$ $\mathrm{HClO}_{4}-\mathrm{H}_{2} \mathrm{SO}_{4}$ followed by another 6 designs of experiments where the combination $\mathrm{HClO}_{4}-\mathrm{H}_{2} \mathrm{SO}_{4}-\mathrm{HNO}_{3}$ was the most accurate according to the records. Then, new proportions were evaluated: 3: 4: 3; 5: 2: 6 and 7: 1: 15, from which the third ratio used reached acceptable results by means of the third being the total re-suspension of the wet salts by means of $\mathrm{HNO}_{3}$ (final evaluation: $1-8 \mathrm{~mL}$ ). When more than $5 \mathrm{~mL} \mathrm{HNO}_{3}$ was used, there were no statistically significant differences $(\mathrm{p}<0.05)$ when compared to the certified values. Quantification was performed by inductively coupled plasma atomic emission spectrometry with the selected tested acid digestion and with the biomodel Gambusia punctata (Poey, 1854) under differential contamination conditions.

Keywords: acid digestion - biomonitors - metals - target organs 


\section{RESUMEN}

El propósito del estudio fue optimizar una mezcla ácida para la bioextracción ecotoxicológica de metales en órganos diana. Desde junio del 2010 hasta junio del 2017 fueron seleccionaron seis tipos de ácidos: $\mathrm{HF}, \mathrm{HCl}, \mathrm{HNO}_{3}, \mathrm{HClO}_{4}$, $\mathrm{H}_{2} \mathrm{SO}_{4}$ y $\mathrm{H}_{3} \mathrm{PO}_{4}$ diseñándose 15 combinaciones (dos tipos de ácidos) y 17 combinaciones (tres tipos de ácidos) para comparar sus resultados con los valores patrones de referencias certificadas sobre peces no expuestos a contaminación por metales: DOLT-3 ("Dogfish Liver", hígado de tiburón) y DORM-2 ("Dogfish Muscle", músculo de tiburón). Los registros más próximos correspondieron a la mezcla: $\mathrm{HNO}_{3}-\mathrm{HClO}_{4}-\mathrm{H}_{2} \mathrm{SO}_{4}$ realizándose luego, otros 6 diseños de experimentos donde la combinación $\mathrm{HClO}_{4}-\mathrm{H}_{2} \mathrm{SO}_{4}-\mathrm{HNO}_{3}$ fue la más precisa según los registros. Después, nuevas proporciones fueron evaluadas: 3:4:3; 5:2:6 y 7:1:15, alcanzándose aceptables resultados mediante la tercera siendo la re-suspensión total de las sales húmedas mediante el $\mathrm{HNO}_{3}$ (valoración final: 1-8mL). Cuando se usó más de $5 \mathrm{~mL}$ de $\mathrm{HNO}_{3}$, no se observó diferencias estadísticamente significativas $(\mathrm{p}<0,05)$ con los valores certificados. La cuantificación se realizó por espectrometría de emisión atómica con plasma inductivamente acoplado con la digestión ácida testeada y con el biomodelo Gambusia punctata (Poey, 1854) bajo condiciones diferenciales de contaminación.

Palabras clave: biomonitores - digestión ácida - metales - órganos diana

\section{INTRODUCCIÓN}

Algunos parámetros físico-químicos de calidad del agua condicionan a la biodisponibilidad de metales (Moreno et al., 2018); y por ende, acumulación en tejidos biológicos (Azaman et al., 2015; Dalzochio \& Gehlen, 2016; Alegre et al., 2018). Los peces muestran evidencias sobre la exposición a metales en el medio natural (Uren et al., 2013) donde su tolerancia, representa una respuesta adaptativa (Hamilton et al., 2016) aunque, resulta necesario seleccionar determinada mezcla de ácido que asegure la extracción de metales para la toma de decisiones ambientales. Entre las incertidumbres analíticas está el reconocimiento sobre la proporción ácida más favorable que permita predecir el riesgo ecotoxicológico. El propósito del estudio fue optimizar una mezcla ácida para la bioextracción ecotoxicológica de metales en órganos diana.

\section{MATERIAL Y MÉTODOS}

El estudio se realizó en el Laboratorio de Minerales de la Empresa Geominera Oriente, Santiago de Cuba-Cuba durante el 2017. Fueron probadas, combinaciones de dos y tres mezcla de ácidos $\left(\mathrm{HF}, \mathrm{HCl}, \mathrm{HNO}_{3}, \mathrm{HClO}_{4}, \mathrm{H}_{2} \mathrm{SO}_{4}\right.$ y $\mathrm{H}_{3} \mathrm{PO}_{4}$ ) para la bioextracción del cadmio coincidiendo con otros estudios (Akinyele \& Shokunbi, 2015). La confiabilidad analítica fue mediante el uso de dos muestras de referencias certificadas: DOLT-3 ("Dogfish Liver", hígado de tiburón) y DORM-2 ("Dogfish Muscle", músculo de tiburón) (Lavilla et al., 2008).

Diferentes proporciones se probaron de acuerdo a, la mezcla ácida que resultó seleccionada y luego, en el modelo ecotoxicológico Gambusia punctata (Poey 1854) (Poeciliidae) se cuantificó mediante espectrometría de emisión atómica por plasma inductivamente acoplado con vista axial: ICP-AES (Galvão et al., 2016), concentraciones de cadmio en las branquias e hígado.

Se utilizó el programa estadístico Statgraphics Centurion 18 para el tratamiento de los datos. La comparación se realizó mediante el análisis de varianza donde el contraste de la homogeneidad de los grupos fue mediante la prueba de Bonferroni considerándose significativos los resultados cuando $\mathrm{p}<0,05$.

Aspectos éticos: los autores señalan que se cumplieron todos los aspectos éticos nacionales e internacionales.

\section{RESULTADOS}

La tabla 1 muestra, combinaciones de mezclas ácidas para bioextraer metales. 
Tabla 1. Combinaciones de mezclas de ácidos para la bioextracción de metales

\begin{tabular}{ccc}
\hline $\mathrm{N}^{\circ}$ & Mezcla de dos ácidos & Mezcla de tres ácidos \\
\hline 1 & $\mathrm{HF}-\mathrm{HCl}$ & $\mathrm{HF}-\mathrm{HCl}-\mathrm{HNO}_{3}$ \\
2 & $\mathrm{HF}-\mathrm{HNO}_{3}$ & $\mathrm{HF}-\mathrm{HCl}-\mathrm{HClO}_{4}$ \\
3 & $\mathrm{HF}-\mathrm{HClO}_{4}$ & $\mathrm{HF}-\mathrm{HCl}-\mathrm{H}_{2} \mathrm{SO}_{4}$ \\
4 & $\mathrm{HF}-\mathrm{H}_{2} \mathrm{SO}_{4}$ & $\mathrm{HF}-\mathrm{HCl}-\mathrm{H}_{3} \mathrm{PO}_{4}$ \\
5 & $\mathrm{HF}-\mathrm{H}_{3} \mathrm{PO}_{4}$ & $\mathrm{HF}-\mathrm{HNO}_{3}-\mathrm{HClO}_{4}$ \\
6 & $\mathrm{HCl}-\mathrm{HNO}_{3}$ & $\mathrm{HF}-\mathrm{HNO}_{3}-\mathrm{H}_{2} \mathrm{SO}_{4}$ \\
7 & $\mathrm{HCl}-\mathrm{HClO}_{4}$ & $\mathrm{HF}-\mathrm{HNO}_{3}-\mathrm{H}_{3} \mathrm{PO}_{4}$ \\
8 & $\mathrm{HCl}-\mathrm{H}_{2} \mathrm{SO}_{4}$ & $\mathrm{HF}-\mathrm{HClO}_{4}-\mathrm{H}_{2} \mathrm{SO}_{4}$ \\
9 & $\mathrm{HCl}-\mathrm{H}_{3} \mathrm{PO}_{4}$ & $\mathrm{HF}-\mathrm{HClO}_{4}-\mathrm{H}_{2} \mathrm{SO}_{4}$ \\
10 & $\mathrm{HNO}_{3}-\mathrm{HClO}_{4}$ & $\mathrm{HF}-\mathrm{HClO}_{4}-\mathrm{H}_{3} \mathrm{PO}_{4}$ \\
11 & $\mathrm{HNO}_{3}-\mathrm{H}_{2} \mathrm{SO}_{4}$ & $\mathrm{HF}-\mathrm{H}_{2} \mathrm{SO}_{4}-\mathrm{H}_{3} \mathrm{PO}_{4}$ \\
12 & $\mathrm{HNO}_{3}-\mathrm{H}_{3} \mathrm{PO}_{4}$ & $\mathrm{HCl}-\mathrm{HNO}_{3}-\mathrm{HClO}_{4}$ \\
13 & $\mathrm{HClO}_{4}-\mathrm{H}_{2} \mathrm{SO}_{4}$ & $\mathrm{HCl}-\mathrm{HNO}_{3}-\mathrm{H}_{2} \mathrm{SO}_{4}$ \\
14 & $\mathrm{HClO}_{4}-\mathrm{H}_{3} \mathrm{PO}_{4}$ & $\mathrm{HCl}-\mathrm{HNO}_{3}-\mathrm{H}_{3} \mathrm{PO}_{4}$ \\
15 & $\mathrm{H}_{2} \mathrm{SO}_{4}-\mathrm{H}_{3} \mathrm{PO}_{4}$ & $\mathrm{HNO}-\mathrm{HClO}_{4}-\mathrm{H}_{2} \mathrm{SO}_{4}$ \\
16 & & $\mathrm{HNO}_{3}-\mathrm{HClO}_{4}-\mathrm{H}_{3} \mathrm{PO}_{4}$ \\
17 & & $\mathrm{HClO}_{4}-\mathrm{H}_{2} \mathrm{SO}_{4}-\mathrm{H}_{3} \mathrm{PO}_{4}$ \\
\hline
\end{tabular}

La tabla 2 muestra los valores certificados de las muestras de referencias donde se aplicaron las combinaciones de mezclas de ácido para la selección más eficiente.

Tabla 2. Valor certificado de las muestras de referencias $\left(\mathrm{mg} \cdot \mathrm{Kg}^{-1}\right)$

\begin{tabular}{ccc}
\hline Metal & \multicolumn{2}{c}{ Referencias } \\
\hline Cadmio & DOLT-3 (Dogfish Liver) & DORM-2 (Dogfish Muscle) \\
& Replicas 11 & Replicas 7 \\
& $19,04 \pm 0,6$ & $0,043 \pm 0,01$ \\
\hline
\end{tabular}

La tabla 3 muestra el análisis de la varianza (para las dos muestras de referencias) sobre la combinación de mezcla ácida $\mathrm{HClO}_{4}-\mathrm{H}_{2} \mathrm{SO}_{4}-\mathrm{HNO}_{3}$ que resultó significativa.

Tabla 3. Análisis de la varianza / mezcla de ácidos / muestras de referencias

\begin{tabular}{ccccccc}
\hline Referencia & Fuente & $\begin{array}{c}\text { Suma de } \\
\text { Cuadrados }\end{array}$ & gl & Cuadrado medio & F-Ratio & P-Value \\
& Between groups & 16,24 & 5 & 3,24 & 91,94 & 0,0000 \\
\multirow{2}{*}{ DOLT-3 } & Within groups & 0,42 & 12 & 0,35 & & \\
& Total (Corr.) & 16,67 & 17 & & & \\
& Between groups & 0,0001 & 5 & 0,00002 & 8,24 & 0,001 \\
\multirow{2}{*}{ DORM-2 } & Within groups & 0,00002 & 12 & 0,000002 & & \\
& Total (Corr.) & 0,00013 & 17 & & & \\
\hline
\end{tabular}


La tabla 4 muestra la homogeneidad entre los grupos mediante la prueba de contraste múltiple por Bonferroni.

Tabla 4. Prueba de rango múltiple / Bonferroni. Ref = referencia. Sig = significancia

\begin{tabular}{cccccc}
\hline Ref. & \multicolumn{1}{c}{ Grupos } & Sig. & Ref. & Groups & Sig. \\
\hline & $\mathrm{HNO}_{3}-\mathrm{HClO}_{4}-\mathrm{H}_{2} \mathrm{SO}_{4}$ & a & & $\mathrm{HNO}_{3}-\mathrm{HClO}_{4}-\mathrm{H}_{2} \mathrm{SO}_{4}$ & a \\
& $\mathrm{HNO}_{3}-\mathrm{H}_{2} \mathrm{SO}_{4}-\mathrm{HClO}_{4}$ & b & & $\mathrm{HNO}_{3}-\mathrm{H}_{2} \mathrm{SO}_{4}-\mathrm{HClO}_{4}$ & a \\
& $\mathrm{H}_{2} \mathrm{SO}_{4}-\mathrm{HNO}_{3}-\mathrm{HClO}_{4}$ & c & & $\mathrm{H}_{2} \mathrm{SO}_{4}-\mathrm{HClO}_{4}-\mathrm{HNO}_{3}$ & a \\
& $\mathrm{HClO}_{4}-\mathrm{HNO}_{3}-\mathrm{H}_{2} \mathrm{SO}_{4}$ & c & & $\mathrm{H}_{2} \mathrm{SO}_{4}-\mathrm{HNO}_{3}-\mathrm{HClO}_{4}$ & a \\
\multirow{5}{*}{ DOLT-3 } & $\mathrm{H}_{2} \mathrm{SO}_{4}-\mathrm{HClO}_{4}-\mathrm{HNO}_{3}$ & c & DORM-2 & $\mathrm{HClO}_{4}-\mathrm{HNO}_{3}-\mathrm{H}_{2} \mathrm{SO}_{4}$ & a \\
& $\mathrm{HClO}_{4}-\mathrm{H}_{2} \mathrm{SO}_{4}-\mathrm{HNO}_{3}$ & d & & $\mathrm{HClO}_{4}-\mathrm{H}_{2} \mathrm{SO}_{4}-\mathrm{HNO}_{3}$ & b \\
\hline
\end{tabular}

La tabla 5 muestra de forma comparativa las muestras de referencias y concentraciones de la mezcla de ácido: $\mathrm{HClO}_{4}-$ $\mathrm{H}_{2} \mathrm{SO}_{4}-\mathrm{HNO}_{3}$ según tres proporciones ensayadas

Tabla 5. Concentraciones de la mezcla $\mathrm{HClO}_{4}-\mathrm{H}_{2} \mathrm{SO}_{4}-\mathrm{HNO}_{3} /$ proporciones / referencias

\begin{tabular}{|c|c|c|c|c|}
\hline \multirow{6}{*}{$\begin{array}{l}\text { Proporciones de } \\
\text { ensayo }\end{array}$} & \multicolumn{4}{|c|}{ Referencias } \\
\hline & \multicolumn{2}{|c|}{ DOLT-3 (Dogfish Liver) } & \multicolumn{2}{|c|}{ DORM-2 (Dogfish Muscle) } \\
\hline & \multicolumn{2}{|c|}{ Replicas 11} & \multicolumn{2}{|c|}{ Replicas 7} \\
\hline & \multicolumn{2}{|c|}{$19,04 \pm 0,6$} & \multicolumn{2}{|c|}{$0,043 \pm 0,01$} \\
\hline & \multicolumn{4}{|c|}{ Resuspensión } \\
\hline & 3 & 5 & 3 & 5 \\
\hline $3: 4: 3$ & $16,44 \pm 0,37$ & $17,79 \pm 0,91$ & $0,033 \pm 0,012$ & $0,042 \pm 0,017$ \\
\hline $5: 2: 6$ & $17,29 \pm 0,21$ & $18,68 \pm 0,24$ & $0,035 \pm 0,011$ & $0,041 \pm 0,014$ \\
\hline $7: 1: 15$ & $18,04 \pm 0,42$ & $18,93 \pm 0,37$ & $0,039 \pm 0,006$ & $0,042 \pm 0,008$ \\
\hline
\end{tabular}

En la especie biomonitor $G$. punctata la determinación de Cd fueron: branquias $(0,06 \pm 0,01)$ e hígado $(0,032 \pm 0,03)$.

\section{DISCUSIÓN}

Diversos estudios sobre la determinación de metales pesados en muestras biológicas refieren el uso de la digestión ácida y cuantificación por ICP (Ahmed et al., 2016; Lo et al., 2017; Binder et al., 2018) para estimar el riesgo ambiental cuando estos elementos superan los niveles permisibles en diferentes matrices y luego, pueden acumularse (Ahmed et al., 2015; Bosch et al., 2016; Shaheen et al., 2016).

El cadmio, es uno de esos metales más peligroso por ser carcinogénico (Varol et al., 2017) y donde se requiere su detección temprana; sin embargo, la adecuada proporción ácida para su análisis resulta necesaria. En este estudio, la combinación correspondiente a la mezcla: $\mathrm{HClO}_{4}-$ $\mathrm{H}_{2} \mathrm{SO}_{4}-\mathrm{HNO}_{3}$ fuela más precisa cuyos resultados óptimos según la comparación con los valores de las muestras de referencias certificadas fueron aceptados lo que permitió finalmente, cuantificar al cadmio en las branquias e hígado de la especie $G$. punctata del ecosistema San Juan, Cuba.

\section{REFERENCIAS BIBLIOGRÁFICAS}

Ahmed, M.; Hui, C.Y.; Guo, X. \& Xing, M.Z. 2016. Microwave assisted digestion followed by ICP-MS for determination of trace metals in atmospheric and Lake Ecosystem. Journal of Environmental Sciences, 55:1-10. 
Ahmed, M.K.; Shaheen, N.; Islam, M.S.; Habibullahal-Mamun, M.; Islam, S.; Mohiduzzaman, M. \& Bhattacharjee, L. 2015. Dietary intake of trace elements from highly consumed cultured fish (Labeo rohita, Pangasius pangasius and Oreochromis mossambicus) and human health risk implications in Bangladesh. Chemosphere, 128: 284-292.

Akinyele, I.O. \& Shokunbi, O.S. 2015. Comparative analysis of dry ashing and wet digestion methods for the determination of trace and heavy metals in food samples. Food Chemistry, 173: 682684 .

Alegre, A.; Bonifaz, E.; Lee, S.E.S.; Alvariño, L. \& José Iannacone. 2018. Monitoreo ecotoxicológico de una cuenca en Huancavélica, Perú afectada por metales pesados. - Revista electrónica de Veterinaria, 19: 1-14 http://www.veterinaria. org/revistas/redvet/n050518.html

Azaman, F.; Juahir, H.; Yunus, K.; Azid, A.; Kamarudin, M.K.A.K.; Toriman, M.E.; Mustafa, A.D.; Amran, M.A.; Hasnam, N.Ch. \& Saudi, A.S.M. 2015. Heavy metal in fish: analysis and human health-a review. Jurnal Teknologi, 77: 61-69.

Binder, G.A.; Metcalf, R.; Atlas, Z. \& Daniel, K.G. 2018. Evaluation of digestion methods for analysis of trace metals in mammalian tissues and NIST 1577c. Analytical Biochemistry, 543: 37-42.

Bosch, A.C.; O’Neill, B.; Sigge, G.O.; Kerwath, S.E. \& Hoffman, L.C. 2016. Heavy metals inmarine fish meat and consumer health: a review. Journal of the Science of Food and Agriculture, 96: 3248.

Dalzochio, T. \& Gehlen, G. 2016. Confounding factors in biomonitoring using fish. Ecotoxicology and Environmental Contamination, 11: 53-61.

Galvão, N.C.; Almeida, B.M.; Galvão, P.E.; Pinto dos Santos, A.M.; Lago da Silva, R.I. \& Neto, J.H.S. 2016. A review of multivariate designs applied to the optimization of methods based on inductively coupled plasma optical emission spectrometry (ICP OES). Microchemical Journal, 128: 331-346.

Hamilton, P.B.; Cowx, I.G.; Oleksiak, M.F.; Griffiths, A.M.; Grahn, M.; Stevens, J.R.; Carvalho, G.R.;
Nicol, E. \& Tyler, C.R. 2016. Population-level consequences for wild fish exposed to sublethal concentrations of chemicals - a critical review. Fish and Fisheries, 17: 545-566.

Lavilla, I.; Vilas, P. \& Bendicho, C. 2008. Fast determination of arsenic, selenium, nickel and vanadium in fish and shellfish by electrothermal atomic absorption spectrometry following ultrasound-assisted extraction. Food Chemistry, 106: 403-409.

Lo, D.Ma.G.; Galvano, F.; Dugo, G.; D’ascenzi, C.; Macaluso, A.; Vella, A.; Giangrosso, G.; Cammilleri, G. \& Ferrantelli, V. 2018. Toxic metal levels in cocoa powder and chocolate by ICP-MS method after microwave-assisted digestion. Food Chemistry, 245:1163-1168.

Moreno, A.S.A.; Gomes, C.P. \& Bianchini, A. 2018. Metal accumulation and expression of genes encoding for metallothionein and copper transporters in a chronically exposed wild population of the fish Hyphessobrycon luetkenii. Comparative Biochemistry and Physiology, 211:25-31.

Shaheen, N.; Ahmed, M.K.; Islam, M.S.; Habibullah-AlMamun, M.; Tukun, A.B.; Islam, S. \& Rahim, A.T.M.A. 2016. Health risk assessment of trace elements via dietary intake of non-piscine protein source foodstuffs (meat, milk and egg) in Bangladesh. Environmental Science and Pollution Research, 23: 7794-7806.

Uren, W.T.M.; Bury, N.; van Aerle, R. \& Santos, E.M. 2013. Global transcriptome profiling reveals molecular mechanisms of metal tolerance in a chronically exposed wild population of brown trout. Environmental Science \& Technology, 47: 8869-8877.

Varol, M.; Kaya, K.G. \& Alp, A. 2017. Heavy metal and arsenic concentrations in rainbow trout (Oncorhynchus mykiss) farmed in a dam reservoir on the Firat (Euphrates) River: risk-based consumption advisories. Science of the Total Environment, 599-600: 1288-1296.

Received December 6, 2018.

Accepted December 31, 2018. 